\title{
FHIT promoter methylation status, low protein and high mRNA levels in patients with non-small cell lung cancer
}

\author{
KAROLINA H. CZARNECKA ${ }^{1}$, MONIKA MIGDALSKA-SĘK ${ }^{1}$, DARIA DOMAŃSKA ${ }^{1}$, \\ DOROTA PASTUSZAK-LEWANDOSKA ${ }^{1}$, AGATA DUTKOWSKA ${ }^{2}$, JACEK KORDIAK $^{3}$, EWA NAWROT ${ }^{1}$, \\ JUSTYNA KISZAŁKIEWICZ ${ }^{1}$, ADAM ANTCZAK ${ }^{2}$ and EWA BRZEZIAŃSKA-LASOTA ${ }^{1}$ \\ Departments of ${ }^{1}$ Molecular Bases of Medicine, ${ }^{2}$ General and Oncological Pulmonology, \\ Medical University of Lodz, ${ }^{3}$ Department of Chest Surgery, General and Oncological Surgery \\ University Hospital No. 2, Medical University of Lodz, Lodz, Poland
}

Received April 4, 2016; Accepted May 12, 2016

DOI: $10.3892 /$ ijo.2016.3610

\begin{abstract}
FHIT is a tumor suppressor gene that is frequently silenced in non-small cell lung cancer (NSCLC) and also in preneoplastic lesions. Promoter hypermethylation was previously observed in NSCLC, and its epigenetic silencing, observed on mRNA or protein level, was proposed to predict NSCLC outcome. In the present study we evaluated the relationship between FHIT expression on mRNA level and promoter methylation, or immunoexpression level. The aim of this study was to analyze the usefulness of FHIT as early differentiating biomarker in NSCLC patients. Lung tissue specimens were obtained from 59 patients with diagnosed NSCLC (SCC=34, $\mathrm{AC}=20, \mathrm{LCC}=5$ ). FHIT promoter methylation was assessed in methylation-specific PCR. Relative expression analysis of FHIT was performed in real-time PCR (qPCR) and protein immunoexpression by ELISA assay. Significant differences in FHIT expression between NSCLC histopathological groups (SCC, AC, LCC) were observed ( $\mathrm{p}=0.000009)$, with the lowest level in SCC. FHIT expression was significantly higher $(\mathrm{p}=0.034)$ in men vs. women. Methylated FHIT alleles were present both in NSCLC and control specimens. Mean MI value was higher in control tissue vs. neoplasm, and in men vs. women and it increased with patient age. Significant increase in MI level was observed in N0 group vs. N1 and N2, according to the TNM staging $(\mathrm{p}=0.0073)$. Differences in FHIT expression levels between AC, LCC and SCC indicated the usefulness of this gene as a diagnostic marker for NSCLC subtype differentiation. FHIT promoter hypermethylation both in cancer and control tissue indicated the presence of epigenetic alterations in early stage of NSCLC development.
\end{abstract}

Correspondence to: Dr Karolina H. Czarnecka, Department of Molecular Bases of Medicine, Medical University of Lodz, 51 Pomorska St., 92-213 Lodz, Poland

E-mail: karolina.czarnecka@umed.lodz.pl

Key words: FHIT, gene expression, promoter methylation, epigenetic mechanisms, lung cancer, non-small cell lung cancer, tobacco smoking
Differences in gene promoter methylation between cancer patients with and without node infiltration might be considered as a prognostic marker. Significantly lower FHIT protein immunoexpression was revealed in the group with long and intense history of smoking assessed as PYs (PY $<40$ vs. $\mathrm{PY} \geq 40$, $\mathrm{p}=0.01)$. These results suggest the need of further study on FHIT as a potential biomarker.

\section{Introduction}

Lung cancer is one of the most prevalent cancers (1.6 million new cases yearly) and one of the most common cause of deaths (more than million per year) with the mortality to incidence ratio 0.86 . Lung cancer stands for $21.2 \%$ of all diagnosed cancers in men, and $8.6 \%$ among women (after breast and colorectal cancers) $(1,2)$. In clinical classification two main types of lung cancer are distinguished: small cell lung cancer (SCLC) and non-small cell lung cancer (NSCLC) accounting for almost $80 \%$ of lung cancers (3). The three main NSCLC subtypes are squamous cell carcinoma (SSC), adenocarcinoma (AC) and large cell cancer (LCC) (4). Late detection of lung tumor (AJCC stage III /IV) drastically reduces the chance for a cure; the 5-year survival rate is $\sim 6.6 \%$ (2). Detection of lung cancer in stage I increase the survival up to $83 \%$ (5). For this reason, it is important to search for candidate biomarkers, which will enable to recognize NSCLC on early stage and will help to distinguish its subtypes.

Tumor suppressor genes (TSG) are potential cancer markers because their expression in tumor tissues is suppressed or lost. Loss of function can occur as an effect of genetic instability (inactivating mutations, loss of heterozygosity) or by the promoter region hypermethylation (epigenetic mechanism). In lung cancer TSG inactivation frequently occurs in critical regions on $3 \mathrm{p}$, such as $3 \mathrm{p} 21$ covering the loci of RASSF1A, $R A R B, M L H 1$ (6). One of the potential biomarker is FHIT located in 3p14.2 described as FRA3B fragile site, the region frequently altered in lung carcinogenesis. FHIT (member of the histidine triad gene family) encodes a diadenosine 5',5'"-P1,P3-triphosphate hydrolase involved in purine metabolism. FHIT protein is homologous to Ap4A hydrolase from the yeast Schizosaccharomyces pombe and also exhibits 
Ap3A activity in enzymatic assays (7). FHIT inhibits the serine/threonine kinase Akt, a key effector in PI3K pathway, promoting survival and cell growth in response to extracellular signals (8). The TSG function of this gene is reflected by regulation of programmed cell death and suppression of tumor metastasis (8). FHIT protein also plays a role in the modulation of response to DNA damage, for example, preventing the replication of stress-induced DNA damage. FHIT interacts with $\mathrm{C}$-terminal domain of $\beta$-catenin, inhibiting the Wnt signaling pathway and its target genes, including cyclin D1, MMP14 and survivin (9). FHIT protein with Chk1 kinase plays important role in $\mathrm{S}$ phase checkpoint. Introduction of a wild-type FHIT gene suppresses tumorigenicity and FHIT transfection in 'FHIT-lacking' human cancer cells appears to induce apoptosis and inhibit cell growth $(10,11)$. Several investigators have shown that loss of FHIT function in preneoplastic lesions can lead to the accumulation of DNA damage and cell transformation; therefore it is defined as the guardian of the preneoplastic genome (12-14).

Aberrant FHIT expression caused by truncated transcripts or promoter region hypermethylation has been found in esophageal, stomach, and colon carcinomas $(7,15)$. Lack of FHIT expression in several studies was demonstrated to have impact on tumor aggressiveness (16). In addition, decreased FHIT expression present in preneoplastic lesions of the lung has been proposed to predict NSCLC outcome (12). LOH-dependent FHIT decreased expression have been linked with high proliferation and low apoptotic index in tumor cells, particularly in SCC (17). It has also been proven that co-hypermethylation of p16 and FHIT genes in early stage of NSCLC is poor prognostic factor and can confer cisplatin resistance in NSCLC cells (18).

The aim of this study was to assess the relationship between FHIT gene promoter methylation level and FHIT gene expression, both in lung cancer tissue and macroscopically unchanged tissue from the operational margin. FHIT protein expression level was also evaluated. The obtained results were correlated with the clinical features of patients, tobacco addiction and histopathological characteristics of lung tumors.

\section{Materials and methods}

Clinical characterization of patients and the NSCLC tissue samples. The study received the approval of the Ethics Committee of the Medical University of Lodz, Poland, agreement no. RNN/140/10/KE. All patients were informed and written consent was obtained from each patient.

The lung tissues were obtained from 65 patients who underwent lobectomy or pneumonectomy between July 2010 and March 2013 in the Department of Thoracic Surgery, General and Oncologic Surgery, Medical University of Lodz, Poland. Patients selected for the study had primary tumors and were preoperatively cytologically and histopathologically assessed. Patients did not undergo chemo- or radiotherapy treatment prior to the surgery. The resected tumors were post-operatively subjected to the histopathological analysis. Based on the histopathological results, the NSCLC diagnoses were confirmed for 59 patients, and those patients qualified for further studies. NSCLC samples in the histopathological evaluation were classified as: squamous cell carcinoma (SCC,
Table I. Patient profile and tumor characteristics.

\begin{tabular}{lcc}
\hline Characteristics & Mean age \pm SD & No. of patient \\
\hline Gender & & \\
Women & $63.08 \pm 7.820$ & $24(40.7 \%)$ \\
Men & $65.78 \pm 7.315$ & $35(59.3 \%)$ \\
NSCLC histopathological verification & \\
SCC & $67.46 \pm 6.13$ & $34(58 \%)$ \\
AC & $65.93 \pm 5.13$ & $20(34.4 \%)$ \\
LCC & $60.91 \pm 3.54$ & $5(8.6 \%)$
\end{tabular}

Tobacco addiction and consumption

Current smokers

$31(53.5 \%)$

Former smokers

$23(43 \%)$

Non-smokers

$4(6.75 \%)$

Pack Years $^{\mathrm{a}}$ (PYs)

$<40$ PYs

$26(48 \%)$

$\geq 40$ PYs

$28(52 \%)$

Lung cancer staging

AJCC Staging ${ }^{\mathrm{b}}$

IA/IB

$14(25 \%)$

IIA/IIB

$23(41 \%)$

IIIA/IIIB

$18(32 \%)$

pTNM staging

pT1

$15(27 \%)$

pT2

$23(41 \%)$

pT3/pT4

$17(30 \%)$

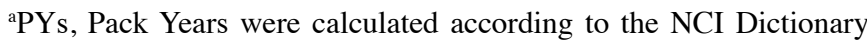
of Cancer Terms: 1 Pack Year is equal to 20 cigarettes smoked per day for one year (http://www.cancer.gov/dictionary?CdrID=306510). bhttps://cancerstaging.org/references-tools/quickreferences/ Documents/LungMedium.pdf (19).

$\mathrm{n}=34$ ), adenocarcinoma ( $\mathrm{AC}, \mathrm{n}=20$ ), and large cell carcinoma (LCC, $\mathrm{n}=5$ ). The tumor samples were classified according to the AJCC staging (19) as well as TNM classification (pTNM) post-operative tumor node metastasis classification according to the WHO Histological Typing of Lung Tumors). The study group comprised of 24 women and 35 men. The smoking history was obtained from each patient. Detailed clinicopathological information on NSCLC patients is presented in Table I.

For the study purposes a pair of lung tissue samples was collected from each patient: from the center of the lesion and from the operational margin (obtained from the most distant site from the resected lesion) - the macroscopically unchanged lung tissue, that served as control tissue. The resected lung tissues (100-150 mg) were immediately placed in RNA stabilizing buffer (RNAlater ${ }^{\circledR}$, Qiagen, Hilden, Germany), cut into smaller parts and stored in $-80^{\circ} \mathrm{C}$ until further use.

Genomic DNA and total RNA isolation. Genomic DNA and total RNA from NSCLC samples and macroscopically unchanged lung tissues were isolated using the column 
Table II. MS-PCR primer sequences and expected product length.

Primer sequence $\left(5^{\prime}-3^{\prime}\right)$

\begin{tabular}{ll} 
Meth F & AAAAGAAATTTAGTTAGTGGGAAGTC \\
Meth R & AAAAAAATTTAAAACATAAATCGCA \\
Unmeth F & AGAAATTTAGTTAGTGGGAAGTTGT \\
Unmeth R & AAAAAAATTTAAAACATAAATCACA \\
\hline
\end{tabular}

Meth, Methylated; Unmeth, Unmethylated; F, forward; R, reverse. Methylated product length, $170 \mathrm{bp}$; unmethylated product length, 167 bp. bp, base pairs.

methods, QIAamp DNA Mini kit (Qiagen) and Universal RNA Purification kit (Eurx Ltd., Gdansk, Poland), according to the manufacturer's protocol. After the isolation, quality and quantity of DNA and RNA was spectrophotometrically assessed (BioPhotometer ${ }^{\mathrm{TM}}$ Plus; Eppendorf, Hamburg, Germany). For further analysis only high quality DNA samples with a $260 / 280 \mathrm{~nm}$ ratio in the range of 1.8-2.0 and DNA concentration over $50 \mathrm{ng} / \mu 1$ were used. RNA was additionally submitted to qualitative and quantitative assessment in automated capillary electrophoresis on Agilent 2100 Bioanalyzer (Agilent Technologies, Santa Clara, CA, USA) using RNA 6000 Pico/Nano LabChip kit (Agilent Technologies) in order to estimate the $18 \mathrm{~S} / 28 \mathrm{~S}$ rRNA ratio.

Evaluation of FHIT gene promoter methylation. Bisulfite conversion: In order to distinguish methylated from unmethylated cytosine in DNA sequence, the bisulfite conversion reaction was performed (20). The conversion was performed with commercially available kit $\mathrm{CpGenome}{ }^{\mathrm{TM}}$ Turbo Bisulfide Modification kit (Chemicon International, Millipore, USA), according to the manufacturer's protocol. For the reaction with sodium bisulfite $1 \mu \mathrm{g}$ of genomic DNA was used. After conversion, the quality and quantity of DNA was spectrophotometrically assessed at $260 / 280 \mathrm{~nm}$ in biophotometer (BioPhotometer ${ }^{\mathrm{TM}}$ Plus; Eppendorf).

Methylation specific PCR. In order to assess the methylation status of the studied gene, the methylation-specific polymerase chain reaction (MS-PCR) was performed using two pairs of primers (methylated and unmethylated) and bisulfite converted DNA. MS-PCR was performed in a total volume of $12.5 \mu 1$ and the mix contained: $2.5 \mu \mathrm{M}$ dNTPs mix, $2.5 \mu \mathrm{M} \mathrm{MgCl}_{2}$, Hot Start AmpliTaq Gold ${ }^{\circledR} 360$ Polymerase (5 U/ $\mu$ l), 10X Universal PCR buffer, nuclease-free water (Applied Biosystems, Foster City, CA, USA), $0.7 \mu \mathrm{M}$ of each primer (Sigma-Aldrich, Poznań, Poland) and 1000 ng of converted DNA. The set of primers for the studied gene was flanking the $1 \mathrm{~kb} 5$ ' region upstream from the translation start point. Two pairs of primers for MS-PCR were designed to amplify the same fragment of FHIT promoter region, according to the criteria described by Feltus et al (21) using the online MethPrimer tool (22). The primer sequences and product length are presented in Table II.

The amplification was conducted in a Thermocycler SureCycler 8800 (Agilent Technologies). MS-PCR conditions were as follows: initial denaturation at $95^{\circ} \mathrm{C}$ for $5 \mathrm{~min}$, followed by 35 cycles involving denaturation at $95^{\circ} \mathrm{C}$ for $45 \mathrm{sec}$, annealing temperature $52.5^{\circ} \mathrm{C}$ for $45 \mathrm{sec}$ and elongation at $72^{\circ} \mathrm{C}$ for $1 \mathrm{~min}$; the final elongation at $72^{\circ} \mathrm{C}$ for $10 \mathrm{~min}$. In order to evaluate the methylation-specific PCR, positive and negative MS-PCR controls were included. In each experiment, blank sample with nuclease-free water instead of DNA was used as a control for PCR contamination. CpGenome universal methylated DNA (enzymatically methylated human male genomic DNA) served as a positive methylation control and CpGenome universal unmethylated DNA (human fetal cell line) was used as a negative control (Chemicon International, Millipore). CpGenome universal methylated and unmethylated DNA were submitted to the bisulfide conversion.

$M S-P C R$ products analysis. In order to analyze the MS-PCR products, the electrophoretic separation was conducted on $2 \%$ agarose gel and visualized in UV transilluminator. Products were also analyzed in automated capillary electrophoresis, using DNA1000 LabChip kit on Agilent 2100 Bioanalyzer (Agilent Technologies). Concentrations (ng) and length of MS-PCR products, $\mathrm{U}$ and M, were spectrophotometrically estimated using DNA size marker (DNA Ladder; Agilent Technologies). Based on the concentrations results the Methylation Index (MI) was assessed for each sample, using the following formula: peak height of methylated products / (peak height of methylated products + peak height of unmethylated product), $\mathrm{MI}=(\mathrm{M}) /(\mathrm{M}+\mathrm{U})$.

Evaluation of FHIT expression. In order to analyze the FHIT gene expression reverse transcription was performed first, using High-Capacity cDNA Reverse Transcription kit (Applied Biosystems). Reverse transcription (RT) master mix contained: 10X RT buffer, 25X dNTP Mix (100 mM), 10X RT Random Primers, MultiScribe ${ }^{\mathrm{TM}}$ Reverse Transcriptase, RNase Inhibitor and nuclease-free water. In RT reaction $100 \mathrm{ng}$ of total RNA was transcribed to complementary DNA (cDNA). RT reaction, in a total volume of $20 \mu \mathrm{l}$, was performed in a Thermocycler SureCycler 8800 (Agilent Technologies). The $\mathrm{RT}$ reaction conditions were as follows: $10 \mathrm{~min}$ at $25^{\circ} \mathrm{C}$, followed by $120 \mathrm{~min}$ at $37^{\circ} \mathrm{C}$, then the samples were heated to $85^{\circ} \mathrm{C}$ for $5 \mathrm{sec}$, and held at $4^{\circ} \mathrm{C}$.

The relative expression of FHIT gene was conducted on Micro Fluidic Cards - TLDA (TaqMan ${ }^{\circledR}$ Low Density Arrays, Applied Biosystems) in Applied Biosystems 7900HT Fast Real-Time PCR System (Applied Biosystems). The qPCR mix contained: $50 \mathrm{ng}$ of cDNA diluted to $50 \mu 1$ in RNAse/DNAse free water, and $50 \mu \mathrm{l}$ TaqMan Universal Master Mix (Applied Biosystems). The selected assays: Hs00179987_m1 for FHIT and Hs00382667_m1 for ESD (esterase D - reference gene) were pre-loaded on the Micro Fluidic Cards. Real-time PCR reaction was processed in program containing $2 \mathrm{~min}$ of initial incubation at $50^{\circ} \mathrm{C}, 10 \mathrm{~min}$ at $94.5^{\circ} \mathrm{C}$ for polymerase activation, followed by 40 cycles of $30 \mathrm{sec}$ denaturation at $97^{\circ} \mathrm{C}$ and $1 \mathrm{~min}$ elongation step at $59.7^{\circ} \mathrm{C}$. The $F H I T$ relative expression was assessed using the comparative delta-delta CT method in TaqMan Relative Quantification Assay software (Applied Biosystems). ESD RNA expression level served as the reference gene to adjust the gene of interest expression value (RQ - relative quantity). Normal lung tissue RNA was 

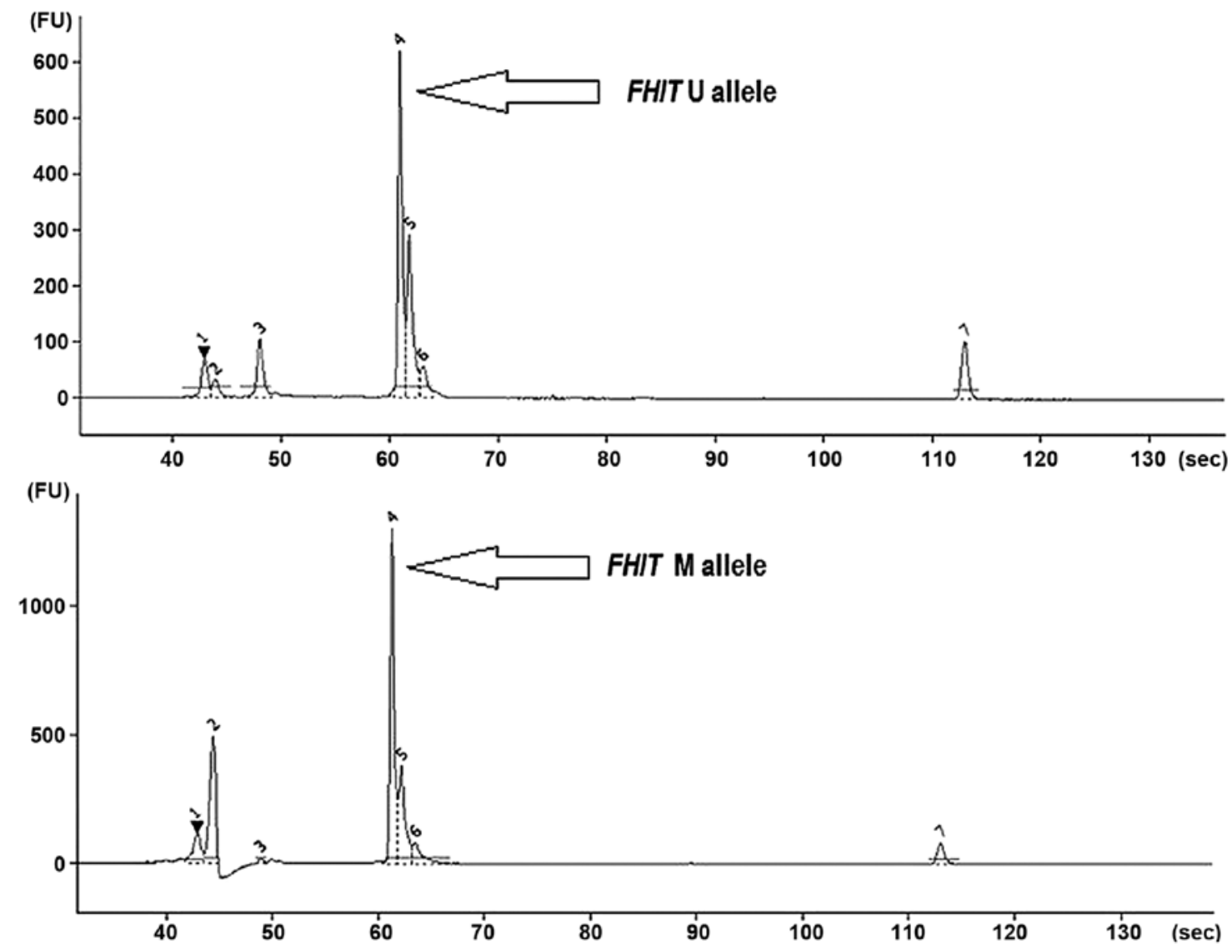

Figure 1. The examples of presence of methylated and unmethylated alleles in cancer tissue of NSCLC patient, SCC subtype (MI=0.79) (Agilent 2100 Bioanalyzer; Agilent Technologies, Santa Clara, CA, USA).

used as a calibrator - Human Lung Total RNA (Ambion ${ }^{\circledR}$, Life Technologies, CA, USA).

Evaluation of FHIT protein expression. Lung tissue samples (10-40 mg) from 51 patients were rinsed in ice-cold PBS buffer (0.01 mol/1, pH 7.0-7.2) and homogenized in $5 \mathrm{ml}$ of PBS buffer. The resulting suspension was subjected to two cycles of freezing and thawing. Then, the homogenates were centrifuged for $5 \mathrm{~min}$ at $5000 \mathrm{x} \mathrm{g}$, the supernatant was removed and the suspension was aliquoted and stored at $-80^{\circ} \mathrm{C}$ until further analysis. FHIT immunoexpression levels in lung tissue homogenates were assessed using ELISA kit for Fragile Histidine Triad Protein (Aviva Systems Biology Corp., San Diego, CA, USA) according to the manufacturer's procedure. The intensity of the final colorimetric reaction, in proportion to the amount of protein bound, was measured in a plate reader (ELx800; BioTek Instruments Inc., Winooski, VT, USA) at the wavelength $450 \mathrm{~nm}$. The obtained results were compared to the standard solutions of known concentrations (100-1000 ng/ml).

Statistical analysis. Statistical analysis was performed using the Statistica for Windows 10.0 software (StatSoft, Cracow, Poland) (v.10). Nonparametrical statistical tests: ANOVA Kruskal-Wallis, Mann-Whitney U test and Spearman's rank correlation coefficient were used in order to evaluate the relationships between gene expression level (RQ), immunoexpression level, gene promoter methylation level (MI) and patient characteristics: age and gender, smoking status (current/former/never smoker), history of smoking measured in pack years (PYs) and clinical features of the tumor (staging according to TNM, AJCC, histopathological NSCLC subtype). The results of relative expression analysis (RQ value), immunoexpression level and gene promoter methylation level (MI) are presented as mean \pm SEM and mean \pm SD values. Statistical significance for all tests were set at $\mathrm{p}<0.05$, and assessed by calculating the p-value.

To identify the parameters associated with FHIT immunoexpression, RQ and MI level stepwise logistic regression analysis with backward selection were performed using patient gender, age, history of smoking measured in pack-years (PY), AJCC and histopathological NSCLC subtype as independent variables selected after exclusion of autocorrelated covariates.

\section{Results}

Evaluation of FHIT gene promoter methylation. The MS-PCR analysis (gel electrophoresis) revealed the presence of methylated (M) and unmethylated (U) FHIT alleles both in NSCLC and control specimens (Fig. 1).

Due to degradations of several DNA samples after bisulfite conversion, methylation levels of 52 NSCLC samples and 31 control specimens (macroscopically unchanged tissues) were assessed in automated capillary electrophoresis (Agilent 2100 Bioanalyzer). The co-presence of $\mathrm{U}$ and $\mathrm{M}$ alleles was the most common, and this was observed for 43 cancer tissue 
Table III. The presence of methylated (M) and unmethylated (U) alleles in histopathological subtypes (SCC, AC, LCC) and paired macroscopically unchanged tissues.

\begin{tabular}{|c|c|c|c|c|c|c|}
\hline NSCLC subtype & Control & $\mathrm{SCC}$ & Control & $\mathrm{AC}$ & Control & $\mathrm{LCC}$ \\
\hline $\mathrm{n}$ & 18 & 29 & 9 & 18 & 5 & 5 \\
\hline $\mathrm{MI}=1$ (only $\mathrm{M}$ alleles) & $5(28 \%)$ & $2(7 \%)$ & 0 & $4(22 \%)$ & $1(20 \%)$ & - \\
\hline $0<\mathrm{MI}<1$ ( $\mathrm{U}$ and $\mathrm{M}$ alleles) & $11(61 \%)$ & $25(86 \%)$ & $7(78 \%)$ & $13(72.5 \%)$ & $4(80 \%)$ & $5(100 \%)$ \\
\hline MI=0 (only U alleles) & $2(11 \%)$ & $2(7 \%)$ & $2(22 \%)$ & $1(5.5 \%)$ & - & - \\
\hline MI value & 0.506 & 0.370 & 0.315 & 0.433 & 0.638 & 0.314 \\
\hline $\mathrm{p}$-value ${ }^{\mathrm{a}}$ & \multicolumn{2}{|c|}{$\mathrm{p}>0.05$} & \multicolumn{2}{|c|}{0.024} & \multicolumn{2}{|c|}{$\mathrm{p}>0.05$} \\
\hline
\end{tabular}

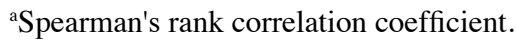

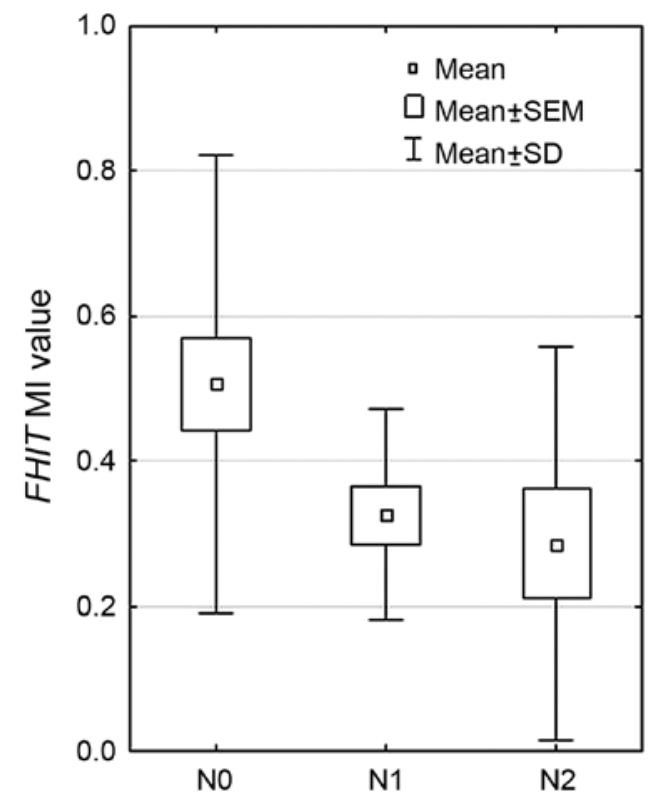

Figure 2. Box and whisker plots representing mean FHIT MI values in the groups according to the 'N' value (pTNM staging).

samples (83\%) and for 21 control specimens (67.5\%). The presence of only $\mathrm{M}$ alleles was detected in 6 cancer tissue samples (11.5\%) and 6 controls (19.5\%). No methylation (only $\mathrm{U}$ alleles) was observed in 4 cancers $(5.5 \%)$ and 3 controls (11\%). Regarding SCC and LCC subtypes, methylated FHIT alleles were present more often in macroscopically unchanged tissue when compared to cancer. Only in AC subtype FHIT methylation was more frequent in cancer tissue, and the difference was statistically significant ( $\mathrm{p}=0.024$, Spearman's rank correlation coefficient). The MI value reflects the observation on the $\mathrm{M}$ and $\mathrm{U}$ allele frequency ratio in cancer and control groups. Methylation level of FHIT (mean MI value) was higher in control tissue $(0.472)$ than in cancer $(0.382)$, but without statistical significance $(\mathrm{p}>0.05$, Spearman's rank correlation coefficient). Data on the presence of $M$ and $U$ alleles in NSCLC subtypes are shown in Table III.

The analysis of MI level between control and cancer tissues in gender groups revealed that higher levels of MI were observed in men vs. women in both tissues: cancer and control (N: 0.416 vs. 0.343 ; C: 0.555 vs. 0.347 , respectively), however
Table IV. FHIT expression levels (mean RQ values) in NSCLC subtypes (SCC, AC, LCC) and paired macroscopically unchanged tissues.

\begin{tabular}{llccr}
\hline & \multicolumn{1}{c}{ Tissue } & $\mathrm{n}$ & Mean RQ & p-value \\
\hline NSCLC group & Cancer & 59 & 1.83 & $>0.05^{\mathrm{a}}$ \\
& Control tissue & 58 & 1.57 & \\
SCC subtype & Cancer & 34 & 1.40 & $>0.05^{\mathrm{a}}$ \\
& Control tissue & 34 & 1.59 & \\
NSCC subtype & Cancer & 25 & 2.41 & $\mathbf{0 . 0 0 1 0 7 3}^{\mathrm{a}}$ \\
(AC + LCC) & Control tissue & 24 & 1.55 & \\
AC subtype & Cancer & 20 & 2.27 & $\mathbf{0 . 0 0 0 1 5 3}^{\mathrm{a}}$ \\
& Control tissue & 19 & 1.61 & \\
LCC subtype & Cancer & 5 & 1.81 & $>0.05^{\mathrm{a}}$ \\
& Control tissue & 5 & 1.30 & \\
\hline
\end{tabular}

${ }^{a}$ Mann Whitney U test.

the differences did not reach statistical significance ( $p>0.05$, Mann-Whitney U test). No statistically significant difference was observed between gender groups, In NSCLC subtypes (SCC, AC, LCC) the differences in MI values according to the gender groups were also evaluated. Mean MI values in men vs. women were higher in SCC (0.382 vs. 0.302$)$ and AC $(0.593$ vs. 0.256$)$, and lower in LCC (0.201 vs. 0.389) ( $>>0.05$, MannWhitney $U$ test). MI values in cancer tissues were increasing with the patient age (in years), however without statistical significance ( $p>0.05$, Spearman's rank correlation coefficient).

Analysis of MI values in groups according to the AJCC classifications demonstrated higher MI in AJCC I (0.481) than in AJCC II (0.326) or AJCC IIIA/B (0.366), (p>0.05, KruskalWallis test). Mean MI values in groups according to TNM staging were similar in pT1 and pT3/4 (0.446 and 0.442, respectively), and lower in pT2 (0.338) (p>0.05, Kruskal-Wallis test). According to the presence of metastasis, it was observed that mean MI value was decreasing with lymph node involvement (pTNM staging, according to the ' $\mathrm{N}$ ' value): the highest MI was observed in patient with N0 (0.526), lower in N1 (0.271) and the lowest in N2 (0.222) $(\mathrm{p}=0.0073$, Kruskal-Wallis test), 
A

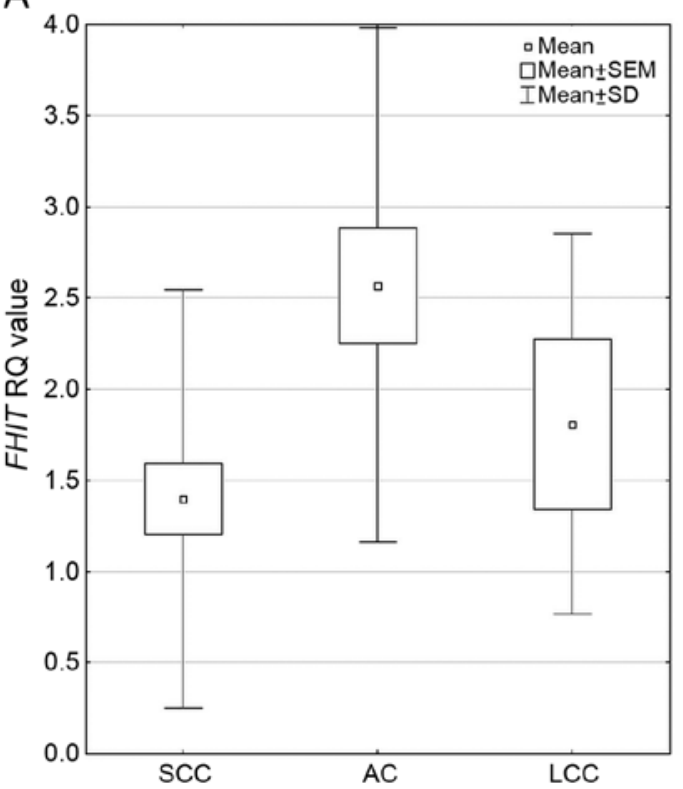

B

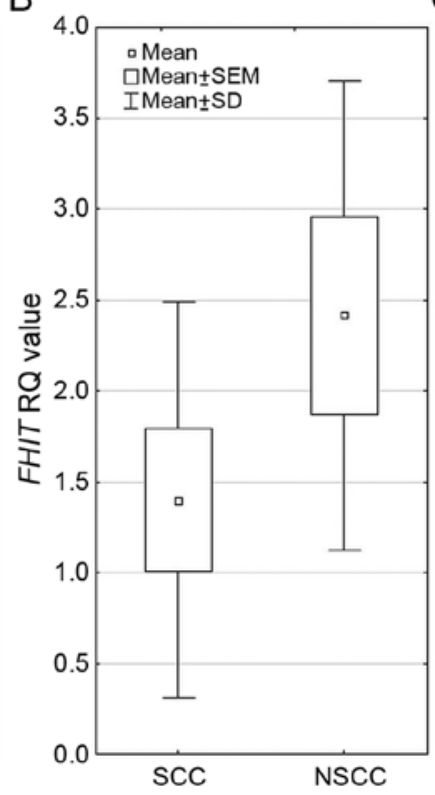

C

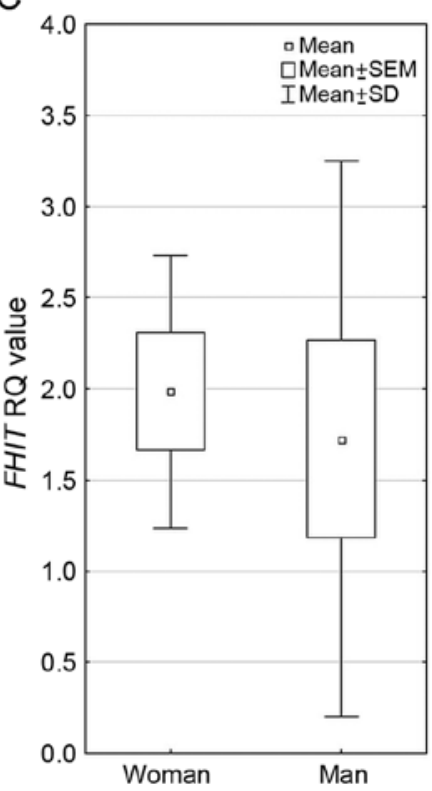

Figure 3. Box and whisker plots representing mean FHIT RQ values in the histopathological subtypes (A and B) of the NSCLC and in gender groups (C).

Table V. FHIT immunoexpression levels assessed by ELISA method and percentage of samples with decreased and without decreased expression level in all studied histopathological NSCLC subtypes.

Samples with:

\begin{tabular}{lcccc}
$\begin{array}{l}\text { Histopathological } \\
\text { NSCLC subtype }\end{array}$ & $\begin{array}{c}\text { Median IE } \\
\text { value } \\
(\mathrm{ng} / \mathrm{ml})\end{array}$ & $\begin{array}{c}\text { Decreased IE value } \\
(\text { range })\end{array}$ & $\begin{array}{c}\text { Decreased IE value } \\
(\%)\end{array}$ & $\begin{array}{c}\text { Non-decreased IE } \\
(\%)\end{array}$ \\
\hline SCC $(\mathrm{n}=30)$ & 326 & $307(118-493)$ & $18(60)$ & $12(40)$ \\
AC $(\mathrm{n}=16)$ & 246 & $262(126-409)$ & $14(88)$ & $2(12)$ \\
LCC $(\mathrm{n}=5)$ & 235 & $275(128-469)$ & $4(80)$ & $1(20)$ \\
Total $(\mathrm{n}=51)$ & 246 & $290(118-493)$ & $36(71)$ & $15(29)$ \\
\hline
\end{tabular}

the results are presented in Fig. 2. Significant differences were observed between N0 vs. N1, and N0 vs. N2 groups ( $\mathrm{p}=0.0113$ and $\mathrm{p}=0.008$, respectively, Mann-Whitney $\mathrm{U}$ test).

No significant relationships were found between FHIT MI values (total NSCLC group, NSCLC subtypes, cancer and control tissues) and smoking history (the length of addiction in years, or tobacco intake in PYs) ( $\mathrm{p}>0.05$, Kruskal-Wallis test, Mann-Whitney U test, followed by Spearman's rank correlation coefficient). Mean MI value was lower in current smokers $(0.345)$ than in former $(0.431)$ or never-smokers $(0.435)$, but in all control tissue groups the MI values were higher than in cancer $(0.381,0.552,0.704$, respectively) ( $p>0.05$, Kruskal-Wallis test).

FHIT gene expression analysis. The expression of FHIT gene, in relation to calibrator sample (RNA from the normal lung tissue), was elevated in all analyzed NSCLC subtypes, and also in macroscopically unchanged tissue. Higher FHIT expression level was detected in the tumor (1.83) than the control (1.57) ( $>0$ 0.05, U-Mann Whitney test). Statistically significant higher RQ levels in cancer when compared to control tissue were observed in AC and NSCC subtypes ( $\mathrm{p}=0.000153$ and $\mathrm{p}=0.001073$, respectively, U-Mann Whitney test). The obtained results are presented in Table IV.

Regarding FHIT expression among the three NSCLC histopathological subtypes, the difference was statistically significant $\mathrm{p}=0.000009$ (Kruskal-Wallis test) and mean RQ value was the highest in AC group. Analysis performed between 2 histological subtypes SCC vs. NSCC (non-squamous cell carcinoma, comprising of $\mathrm{AC}$ and $\mathrm{LCC}$ ) revealed statistically significant increase in FHIT expression in NSCC group ( $\mathrm{p}=0.00001$, U-Mann Whitney test). In gender groups, FHIT expression was significantly higher in women than in men ( 1.984 vs. 1.723 , respectively; $p=0.0351$ U-Mann Whitney test). These results are presented in Fig. 3. With increasing age of patients, the relative expression level of FHIT was decreasing in cancer tissues ( $>0.05$, Spearman's rank correlation coefficient).

According to TNM classification, in total NSCLC group, the RQ value increased with tumor size: pT1 (1.457), pT2 (1.789), pT3/4 (2.25), and similar observation was made in NSCLC subtypes - in SCC and NSCC groups, however the 
Table VI. Multivariate logistic regression model for FHIT immunoexpression coefficients and summary.

\begin{tabular}{lccccccccc}
\hline Factor & $\mathrm{B}$ & Std. Error & Beta & $\mathrm{t}$ & $\mathrm{p}$-value & $\mathrm{R}$ & $\mathrm{R}^{2}$ & Adjusted R square & Std. Error of the estimate \\
\hline $\mathrm{PY}$ & -0.34 & 0.15 & -80.637 & -2.28 & 0.02 & 0.34 & 0.12 & 0.09 & 111.69 \\
\hline
\end{tabular}

FHIT immunoexpression in histopatological subtypes

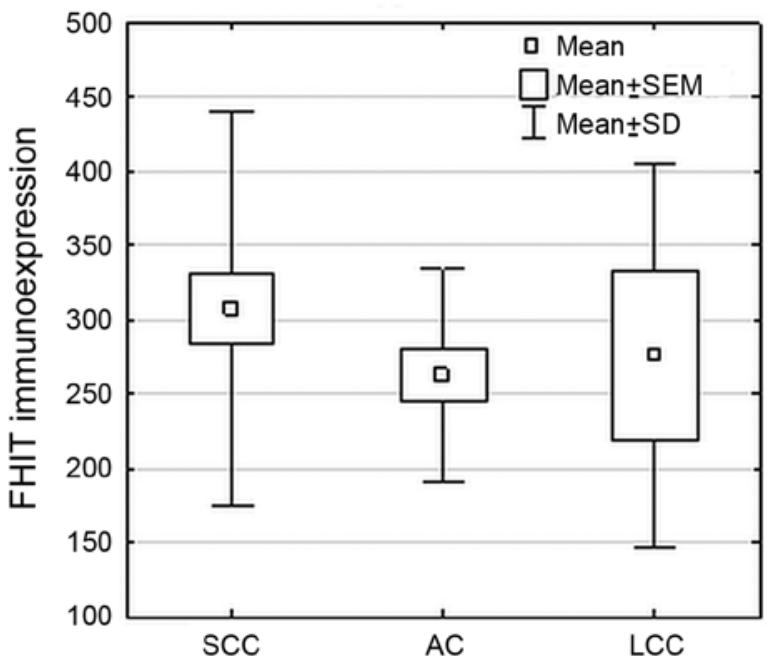

Figure 4. Box-and-whisker plot, representing the FHIT immunoexpression in histopathological subtypes ( $p>0.05$; Mann-Whitney $U$ test) in total NSCLC cohort.

differences were not significant ( $\mathrm{p}>0.05$, Kruskal-Wallis test). In AJCC groups, the mean RQ value was the lowest in AJCC III, and the highest in AJCC II ( $\mathrm{p}>0.05$, Kruskal-Wallis test). In relation to smoking history of patients, FHIT expression was higher in current smokers (1.96), than in former smokers (1.68) and non-smokers (1.70) ( $\mathrm{p}>0.05$, Kruskal-Wallis test). No statistically significant relationships were found between FHIT expression (total NSCLC group, NSCLC subtypes) and smoking history (the length of addiction in years, or tobacco intake in PYs) ( $p>0.05$, Kruskal-Wallis test, Mann-Whitney $\mathrm{U}$ test). Of note, in the group of active smokers with the highest intake in PYs (>45 PYs) the RQ level was the lowest.

FHIT protein expression analysis. The immunoexpression level of FHIT $<350 \mathrm{ng} / \mathrm{ml}$ (in tissue homogenates) was considered as decreased. The decreased immunoexpression was observed in $71 \%$ of all NSCLC samples, and was in the range of $60-88 \%$, depending on the histotype. The results are presented in Table V.

Statistical analysis did not reveal significant differences in FHIT protein expression levels between studied histopathological subtypes (SCC, AC and LCC) ( $p>0.05$; ANOVA Kruskal-Wallis test) or between SCC and NSCC group ( $>0.05$; U Mann-Whitney's test), as shown in Fig. 4.

In the whole cohort of NSCLC patients, statistically significant higher FHIT immunoexpression was revealed in the group of heavy smokers (PYs $\geq 40$ ) (PY $<40$ vs. PY $\geq 40$, $\mathrm{p}=0.01$, Mann-Whitney U test). Such correlation was also

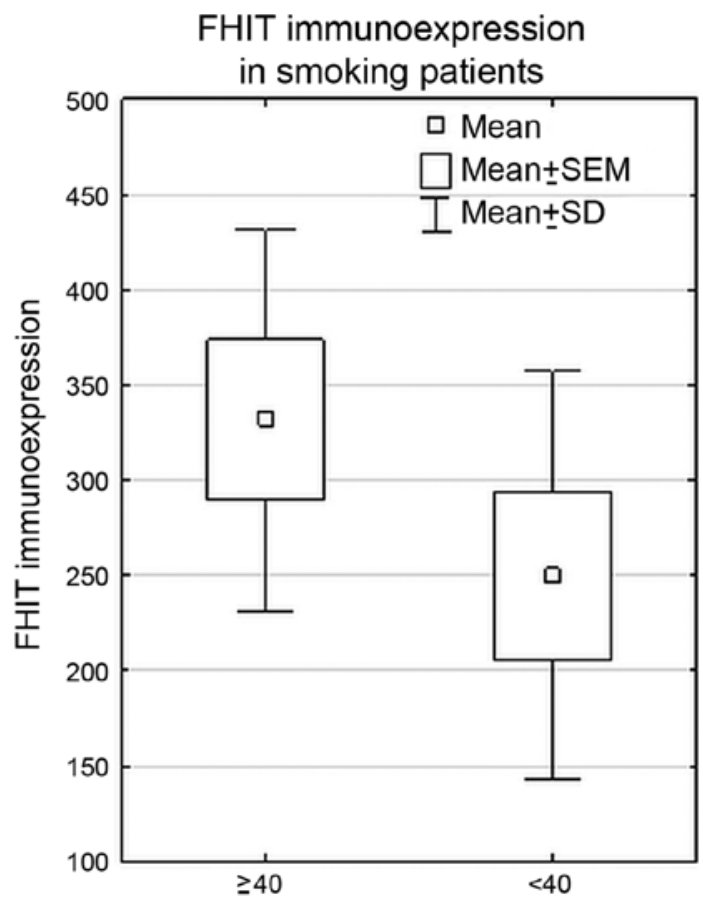

Figure 5. Box-and-whisker plots, representing the FHIT immunoexpression in $\geq 40$ PY and $<40$ PY groups ( $\mathrm{p}=0.01$; Mann-Whitney $\mathrm{U}$ test) in total NSCLC cohort.

found in SCC subtype ( $p=0.01$, Mann-Whitney $U$ test). The results are shown in Fig. 5.

Multivariate stepwise logistic regression analysis with backward selection revealed that higher protein immunoexpression level was correlated with lower value of PYs smoked in a lifetime. This significant correlation was confirmed in multivariate logistic regression model shown in Table VI. The PYs variable explains only $9 \%$ of total immunoexpression variance $\left(\mathrm{R}^{2}=0.09\right)$.

There were no statistically significant correlations between FHIT protein levels and the clinical features of the studied NSCLC patients, i.e., patient age, gender, and status of smoking ( $>0.05$; Mann-Whitney U test, ANOVA Kruskal-Wallis test, followed by Spearman's rank correlation coefficient). Statistical analysis did not reveal any associations between FHIT immunoexpression level and pTNM or AJCC classifications ( $\mathrm{p}>0.05$; ANOVA Kruskal-Wallis test).

Correlations between gene expression and methylation or protein expression values. It was observed that in all analyzed groups the mean RQ values were elevated in cancer when compared to control tissue, and MI was lower in cancer, however we did not find any significant correlations ( $p>0.05$, Spearman's rank correlation coefficient). No significant association between MI and RQ values was found among 
NSCLC subtypes (SCC, AC, LCC), gender and age groups, tobacco addiction or cancer staging ( $p>0.05$, Spearman's rank correlation coefficient). Similarly, there were no statistically significant correlations between FHIT expression and protein immunoexpression among NSCLC subtypes (SCC, AC, LCC), gender and age groups, tobacco addiction or cancer staging ( $\mathrm{p}>0.05$, Spearman's rank correlation coefficient). In addition, there were no statistically significant correlations between FHIT protein immunoexpression and MI levels ( $p>0.05$, Spearman's rank correlation coefficient).

\section{Discussion}

FHIT, the tumor suppressor gene localized on $3 \mathrm{p}$ fragile site (3p14.2), is frequently altered in many human cancers (renal, lung, gastric, lymphomas) (7,12). FHIT expression loss was detected frequently during the early onset of disease progression in cancer $(14,23)$. Loss of function of the FHIT gene can lead to constitutive accumulation of high levels of intracellular diadenosine tetraphosphate and the stimulation of DNA synthesis and proliferation $(25,26)$. Reduction of FHIT expression is consider as poor diagnostic factor correlated with tumor aggressiveness due to the epithelial-mesenchymal transition (EMT) (16,26-28). The EMT is considered as crucial step in the early stage of cancer metastasis. Activation of FHIT gene can enhance the cell ability to enter apoptosis and to inhibit cell growth (29). Several studies have underlined the putative function of FHIT gene as lung cancer biomarker $(12,18)$. In mouse lung cancer model FHIT function was linked with protecting against chemically-induced cancerogenesis (30). In lung cancer cell lines restoration of FHIT expression resulted in induction of apoptosis and tumorigenicity suppression, therefore the gene was proposed as potential agent in targeted gene therapy (11).

In the present study, we assessed FHIT expression on mRNA and protein level, as well as gene promoter methylation. Analysis was performed in primary lung lesions and in macroscopically unchanged lung tissues to deepen the knowledge of potential significance of FHIT as an early diagnostic biomarker. Searching for such biomarkers is very important, especially when lack of effective diagnostic tools at the early stage of the disease can cause up to $85 \%$ mortality rate (in 5 years) (2).

In the previously conducted studies, the presence of FHIT alteration ( $\mathrm{LOH}$, expression alteration) was detected in preneoplastic bronchial lesions (12). Our study is the first one where both mRNA expression level and gene methylation status were analyzed in cancer and macroscopically unchanged lung tissue. We demonstrated that altered gene expression was not only characteristic for cancer, we also observed increased gene expression both in NSCLC tissue and macroscopically unchanged lung tissue from the same patients.

In our study we found elevated FHIT expression in all NSCLC subtypes which is contrary to the results of other groups $(14,23,26,31)$. Also, in our study, FHIT expression level in macroscopically unchanged tissue, regarded as 'normal', although surrounding the primary lesion, was elevated, when compared to calibrator RNA. This can stand for the hypothesis that FHIT activation in lung carcinogenesis process is a response to accumulation of genetic changes in the cells $(12,32)$. The differences in FHIT expression between samples from the same patient could suggest the important role of FHIT gene in a very early stage of lung carcinogenesis.

Also in methylation analysis we demonstrated promoter region hypermethylation in both tissues. This finding is inconsistent with the results of Feng et al, who found no methylation in cancerous or non-cancerous tissues (33). In our study, in case of AC subtype, FHIT promoter methylation status was significantly higher than in normal tissue.

Statistically significant differences in the FHIT expression between histopathological subtypes AC, LCC and SCC could suggest the possibility to deepen the study on the gene as a differentiating marker for NSCLC subtypes. Additionally, it might help in the selection of therapy. Differences between expression in subtypes (the lowest in SCC, the highest in AC) may be under consideration as NSCLC prognostic marker. Unfortunately, these differences between subtypes were not observed in the methylation analysis.

Increased expression of FHIT gene, identified in the current work, would suggest the resulting elevation of its product the FHIT protein. However such result was not confirmed. The analysis of immunoexpression revealed the reduction of FHIT protein level in NSCLCs tissue samples, which was generally consistent with earlier results $(23,25,31,32,34,35)$. We observed FHIT protein reduction in $71 \%$ of NSCLCs, and there was no difference between the histopathological subtypes (88\% AC, $60 \%$ SCC). It is contrary to the findings of other authors, who reported significant loss of protein expression mainly in SCC $(25,34)$. Tomizawa et al (34) described the decreased level of FHIT expression in only $10 \%$ of AC samples in comparison to $86 \%$ of SCCs. The possible reason of the inconsistency between our results and the compared reports could be different material and molecular techniques used to evaluate FHIT immunoexpression. We assessed it in tumor tissue homogenates, while others used paraffin-embedded tissue blocks (17), thus the data cannot be compared reliably. On the other hand, such divergent results suggest poor usage of FHIT immunoexpression as a differentiating marker, that has been recently confirmed in the review article by Lindskog et al (36).

Many studies revealed that loss of FHIT expression or immunoexpression was significantly associated with tumors occurring in heavy smokers $(24,25,32,35,37)$. We have not confirmed this on mRNA level - neither in relation to the length of smoking or to the amount of cigarettes smoked. Interestingly, we found significant differences on protein level: in heavy smokers group FHIT protein level was significantly higher (in total NSCLC group and in SCC group). Sozzi et al (25) obtained the opposite results, the protein expression decreased with heavy smoking.

This discrepancy can be explained by the results of multivariate regression model showing that only $9 \%$ of FHIT immunoexpression variance can be explained by the number of PYs. Such low contribution of smoking history to the FHIT protein level in our study suggest the impact of other, probably more important factors on FHIT protein reduction. Several other possible explanations could be considered, including the method used. In our study we analyzed FHIT immunoexpression with ELISA method in cancer tissue homogenates, not immunohistochemically stained paraffin-embedded tissues. The methodological difference might be also due to the 
contamination of tumor specimens with non-cancerous cells because the tumors analyzed in this study were macrodissected and not microdissected.

In many studies it was confirmed that FHIT activation or enforced expression significantly suppressed metastasis, accompanied by inhibition of EMT $(26,27,28)$. We demonstrated statistically significant differences in gene methylation status according to the node infiltration status (TNM staging) in the group N0 vs. N1 and N2. Lower gene methylation in groups of patients with nodes infiltration could be due to the actions taken by the cell leading to growth inhibition, as FHIT plays role in EMT inhibition. However, these lower methylation levels did not correlate with expression enhancement. In Suh et al study (28), the elevated expressions of FHIT gene and FHIT-dependent miR-30c were proposed as metastasis predictor, as patients with elevated expression had improved metastasis free-survival. In our study the observed difference in FHIT methylation level between patients with and without nodes infiltration could be considered as prognostic marker.

In conclusion, we demonstrated the presence of FHIT promoter methylation both in cancer and control tissue and altered gene expression in both tissue types. It is worth mentioning, that our results give information on methylation level of gene promoter, not only the presence of methylated gene. This can give deeper insight into epigenetic landscape of the lung cancer tissue. The presence of gene promoter hypermethylation both in cancer and control tissue and in different TNM groups suggests early involvement of epigenetic alterations in the development of NSCLC. Differences in FHIT methylation status between NSCLC patients with and without nodes infiltration seems to be considered as prognostic marker. However, these findings do not confirm the observations of other scientist, especially we did not prove the negative correlation between FHIT expression and methylation. Reassuming, the results of our study indicate the value of FHIT gene expression as a differentiating marker of histopathological subtypes of NSCLC. Ambiguous results concerning relationship between FHIT protein level and the amount of cigarettes smoked in a lifetime, suggest unclear impact of smoking on this particular gene. Results of our study indicate that the observed level of FHIT promoter methylation was not enough to suppress gene expression. Lack of negative correlation between FHIT expression and methylation, or positive correlation between gene expression and immunoexpression suggest the role of another molecular mechanisms regulating FHIT expression on mRNA and protein levels in NSCLC patients.

\section{Acknowledgements}

The study was funded by the scientific grant of the Polish National Science Centre, no. UMO-2011/01/B/NZ4/04966.

\section{References}

1. Wojciechowska U, Didkowska J and Zatoński W: Malignant neoplasms. In: Cancer in Poland in 2012. Oncology Centre Marie Curie Institute (ed). Warszawa, pp11-25, 2012.

2. Ferlay J, Shin HR, Bray F, Forman D, Mathers C and Parkin DM: Estimates of worldwide burden of cancer in 2008: GLOBOCAN 2008. Int J Cancer 127: 2893-2917, 2010.

3. Travis WD, Travis LB and Devesa SS: Lung cancer. Cancer 75 (Suppl): 191-202, 1995.
4. Travis WD, Brambilla E, Müller-Hermelink HK and Harris CC (eds): Tumours of the lung. In: WHO Classification Pathology and Genetics of Tumours of the Lung, Pleura, Thymus and Heart. IARC Press, pp9-122, 2004.

5. Kathuria H, Gesthalter Y, Spira A, Brody JS and Steiling K: Updates and controversies in the rapidly evolving field of lung cancer screening, early detection, and chemoprevention. Cancers (Basel) 6: 1157-1179, 2014.

6. Zabarovsky ER, Lerman MI and Minna JD: Tumor suppressor genes on chromosome $3 p$ involved in the pathogenesis of lung and other cancers. Oncogene 21: 6915-6935, 2002.

7. Ohta M, Inoue H, Cotticelli MG, Kastury K, Baffa R, Palazzo J, Siprashvili Z, Mori M, McCue P, Druck T, et al: The FHIT gene, spanning the chromosome 3p14.2 fragile site and renal carcinoma-associated $\mathrm{t}(3 ; 8)$ breakpoint, is abnormal in digestive tract cancers. Cell 84: 587-597, 1996.

8. Semba S, Trapasso F, Fabbri M, McCorkell KA, Volinia S, Druck T, Iliopoulos D, Pekarsky Y, Ishii H, Garrison PN, et al: Fhit modulation of the Akt-survivin pathway in lung cancer cells: Fhit-tyrosine 114 (Y114) is essential. Oncogene 25: 2860-2872, 2006.

9. Weiske J, Albring KF and Huber O: The tumor suppressor Fhit acts as a repressor of beta-catenin transcriptional activity. Proc Natl Acad Sci USA 104: 20344-20349, 2007.

10. Siprashvili Z, Sozzi G, Barnes LD, McCue P, Robinson AK, Eryomin V, Sard L, Tagliabue E, Greco A, Fusetti L, et al: Replacement of Fhit in cancer cells suppresses tumorigenicity. Proc Natl Acad Sci USA 94: 13771-13776, 1997.

11. Roz L, Gramegna M, Ishii H, Croce CM and Sozzi G: Restoration of fragile histidine triad (FHIT) expression induces apoptosis and suppresses tumorigenicity in lung and cervical cancer cell lines. Proc Natl Acad Sci USA 99: 3615-3620, 2002.

12. Fong KM, Biesterveld EJ, Virmani A, Wistuba I, Sekido Y, Bader SA, Ahmadian M, Ong ST, Rassool FV, Zimmerman PV, et al: FHIT and FRA3B 3p14.2 allele loss are common in lung cancer and preneoplastic bronchial lesions and are associated with cancer-related FHIT cDNA splicing aberrations. Cancer Res 57: 2256-2267, 1997.

13. Pichiorri F, Okumura H, Nakamura T, Garrison PN, Gasparini P, Suh SS, Druck T, McCorkell KA, Barnes LD, Croce CM, et al: Correlation of fragile histidine triad (Fhit) protein structural features with effector interactions and biological functions. J Biol Chem 284: 1040-1049, 2009.

14. Saldivar JC, Bene J, Hosseini SA, Miuma S, Horton S, Heerem NA and Huebner K: Characterization of the role of Fhit in suppression of DNA damage. Adv Biol Regul 53: 77-85, 2013.

15. Wang HL, Zhou PY, Liu P and Zhang Y: Abnormal FHIT protein expression may be correlated with poor prognosis in gastric cancer: A meta-analysis. Tumour Biol 35: 6815-6821, 2014.

16. Joannes A, Bonnomet A, Bindels S, Polette M, Gilles C, Burlet H, Cutrona J, Zahm JM, Birembaut P and Nawrocki-Raby B: Fhit regulates invasion of lung tumor cells. Oncogene 29: 1203-1213, 2010.

17. Toledo G, Sola JJ, Lozano MD, Soria E and Pardo J: Loss of FHIT protein expression is related to high proliferation, low apoptosis and worse prognosis in non-small-cell lung cancer. Mod Pathol 17: 440-448, 2004

18. Kim JS, Kim JW, Han J, Shim YM, Park J and Kim DH: Cohypermethylation of p16 and FHIT promoters as a prognostic factor of recurrence in surgically resected stage I non-small cell lung cancer. Cancer Res 66: 4049-4054, 2006.

19. American Joint Committee on Cancer Staging according to the IASLC Staging Project. 7th edition. Cancer, 2010 http:// cancerstaging.org/references-tools/quickreferences/documents/ lungmedium.pdf.

20. Herman JG, Graff JR, Myöhänen S, Nelkin BD and Baylin SB: Methylation-specific PCR: A novel PCR assay for methylation status of CpG islands. Proc Natl Acad Sci USA 93: 9821-9826, 1996.

21. Feltus FA, Lee EK, Costello JF, Plass C and Vertino PM: Predicting aberrant $\mathrm{CpG}$ island methylation. Proc Natl Acad Sci USA 100: 12253-12258, 2003.

22. Li LC and Dahiya R: MethPrimer: Designing primers for methylation PCRs. Bioinformatics 18: 1427-1431, 2002.

23. Sozzi G, Tornielli S, Tagliabue E, Sard L, Pezzella F, Pastorino U, Minoletti F, Pilotti S, Ratcliffe C, Veronese ML, et al: Absence of Fhit protein in primary lung tumors and cell lines with FHIT gene abnormalities. Cancer Res 57: 5207-5212, 1997. 
24. Sozzi G, Sard L, De Gregorio L, Marchetti A, Musso K, Buttitta F, Tornielli S, Pellegrini S, Veronese ML, Manenti G, et al: Association between cigarette smoking and FHIT gene alterations in lung cancer. Cancer Res 57: 2121-2123, 1997.

25. Sozzi G, Pastorino U, Moiraghi L, Tagliabue E, Pezzella F, Ghirelli C, Tornielli S, Sard L, Huebner K, Pierotti MA, et al: Loss of FHIT function in lung cancer and preinvasive bronchial lesions. Cancer Res 58: 5032-5037, 1998.

26. Zhao P, Li XY and Chen LZ: Loss of fragile histidine triad expression and metastasis in breast cancer. Ai Zheng 21: 668-670, 2002 (In Chinese).

27. Bekar A, Ceçener G, Tunca B, Guler G, Egeli U and Tolunay S: Investigation of mutations and expression of the FHIT gene in Turkish patients with brain metastases derived from non-small cell lung cancer. Tumori 93: 604-607, 2007.

28. Suh SS, Yoo JY, Cui R, Kaur B, Huebner K, Lee TK, Aqeilan RI and Croce CM: FHIT suppresses epithelial-mesenchymal transition (EMT) and metastasis in lung cancer through modulation of microRNAs. PLoS Genet 10: e1004652, 2014

29. Song X, Tian Z, Wang S, Peng Z and Feng J: Restoration of fragile histidine triad (FHIT) expression inhibits cell growth and induces apoptosis in cutaneous T-cell lymphoma cell line. Cancer Invest 28: 1019-1023, 2010.

30. Zanesi N, Fidanza V, Fong LY, Mancini R, Druck T, Valtieri M, Rüdiger T, McCue PA, Croce CM and Huebner K: The tumor spectrum in FHIT-deficient mice. Proc Natl Acad Sci USA 98: 10250-10255, 2001.

31. Geradts J, Fong KM, Zimmerman PV and Minna JD: Loss of Fhit expression in non-small-cell lung cancer: Correlation with molecular genetic abnormalities and clinicopathological features. Br J Cancer 82: 1191-1197, 2000.
32. Pavelić K, Krizanac S, Cacev T, Hadzija MP, Radosević S, Crnić I, Levanat S and Kapitanović S: Aberration of FHIT gene is associated with increased tumor proliferation and decreased apoptosis-clinical evidence in lung and head and neck carcinomas. Mol Med 7: 442-453, 2001.

33. Feng Q, Hawes SE, Stern JE, Wiens L, Lu H, Dong ZM, Jordan CD, Kiviat NB and Vesselle H: DNA methylation in tumor and matched normal tissues from non-small cell lung cancer patients. Cancer Epidemiol Biomarkers Prev 17: 645-654, 2008.

34. Tomizawa Y, Nakajima T, Kohno T, Saito R, Yamaguchi N and Yokota J: Clinicopathological significance of Fhit protein expression in stage I non-small cell lung carcinoma. Cancer Res 58: 5478-5483, 1998

35. Tseng JE, Kemp BL, Khuri FR, Kurie JM, Lee JS, Zhou X, Liu D, Hong WK and Mao L: Loss of Fhit is frequent in stage I non-small cell lung cancer and in the lungs of chronic smokers. Cancer Res 59: 4798-4803, 1999.

36. Lindskog $\mathrm{C}$, Edlund $\mathrm{K}$, Mattsson JS and Micke P: Immunohistochemistry-based prognostic biomarkers in NSCLC: Novel findings on the road to clinical use? Expert Rev Mol Diagn 15: 471-490, 2015.

37. Marchetti A, Pellegrini S, Bertacca G, Buttitta F, Gaeta P, Carnicelli V, Nardini V, Griseri P, Chella A, Angeletti CA, et al: FHIT and p53 gene abnormalities in bronchioloalveolar carcinomas. Correlations with clinicopathological data and K-ras mutations. J Pathol 184: 240-246, 1998. 